\title{
Multi-Dimensional Data Aggregation in the Analysis of Self-Similar Processes
}

\author{
M. Poltavtseva* and T. Andreeva ${ }^{\dagger}$ \\ Peter the Great St. Petersburg Polytechnic University, \\ 29 Politechnicheskaya Str, 195251 Saint-Petersburg, RUSSIA
}

\begin{abstract}
Analyzing self-similar processes in various fields requires fast and efficient processing of large amounts of data. The frequency and time scalability of self-similar processes require analysis over multiple time periods. Thus it is necessary to develop effective methods of data aggregation. The paper considers the hierarchical organization of time series and multidimensional aggregation based on a graph. The effectiveness of the proposed aggregation methods and their applicability to the analysis of self-similar processes in various fields are evaluated.
\end{abstract}

AMS Subject Classification: 00A06, 05C05, 05C20

Keywords: mathematics for engineering, trees, directed graphs, self-similar processes, data analysis, big data, data aggregation, hierarchical aggregation, multidimensional aggregation

DOI: https://doi.org/10.33581/1561-4085-2020-23-3-262-269

\section{Introduction}

Self-similar processes are found in a large number of areas: physics, chemistry, information technology, and others. Today, the study of these processes is associated with processing a large amount of data (Big data). Therefore, an important task is to effectively aggregate and prepare data for analysis.

A number of tasks are also characterized by requirements for the speed of self-similar data processing. For example, to detect traffic anomalies [1, 2]. In this case, not only batch systems for processing big data using MapReduce technology are used [3]. For such tasks, one need to use streaming processing modules [4], including special Big data aggregation components [5].

This work is devoted to the development of data aggregation methods for self-similar processes [6], taking into account the need for joint analysis of various time series of data, on the example of multidimensional analysis of network traffic in intrusion detection systems [7].

\footnotetext{
${ }^{*}$ E-mail: poltavtseva@ibks.spbstu.ru

${ }^{\dagger}$ E-mail: atm.nimeria@gmail.com
}

\section{Data aggregation when analyzing self-similar processes}

Informally, a self-similar (fractal) process can be defined as a random process whose statistical characteristics exhibit scaling properties. In mathematics, self-affinity is a feature of a fractal whose pieces are scaled by different amounts in various directions. A compact topological space $\mathrm{X}$ is self-similar if there exists a finite set $\mathrm{S}$ indexing a set of non-surjective homeomorphisms $\left\{f_{a} s \in S\right\}$ for which

$$
X=\bigcup_{s \in S} f_{s}(X)
$$

If $X \subset Y$, then $\mathrm{X}$ self-similar if it is the only nonempty subset of $Y$ such that the equation above holds for $\left\{f_{a} s \in S\right\}$. So,

$$
\mathfrak{L}=\left(X, S,\left\{f_{s}: s \in S\right\}\right)
$$

is a a self-similar structure. The homeomorphisms may be iterated, resulting in an iterated function system. The composition of functions creates the algebraic structure of a monoid. When the set $\mathrm{S}$ has only two elements, the monoid is known as the dyadic monoid. The dyadic monoid can 


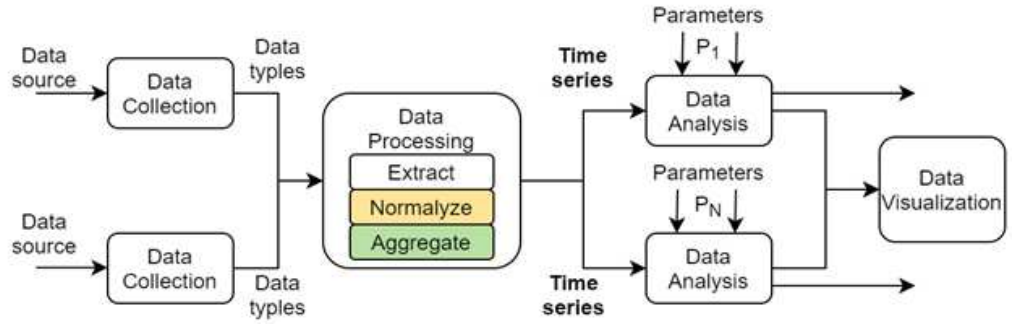

FIG. 1: (color online) Preparing and analyzing data from self-similar processes

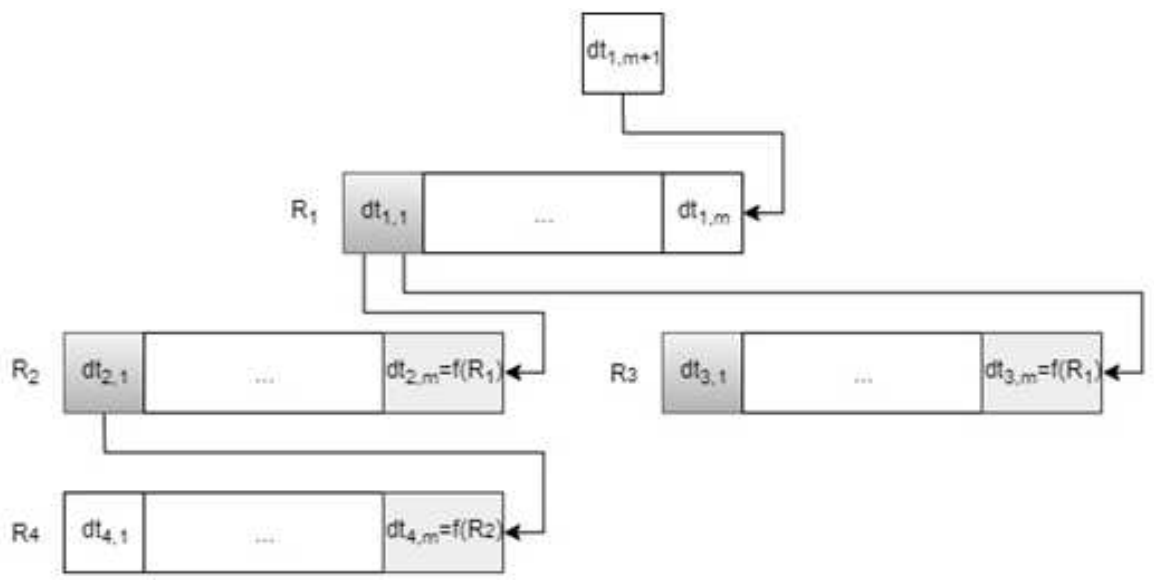

FIG. 2: Hierarchical organization of aggregation time windows

be visualized as an infinite binary tree; more generally, if the set $\mathrm{S}$ has $\mathrm{p}$ elements, then the monoid may be represented as a p-adic tree. When one analyzing data from such structures, they are presented as series. When self-similarity in time-time series is appropriate.

A self-similar process does not significantly change its appearance when viewed at different scales on the time scale. Time series of data are used to analyze self-similar processes. The input data for intrusion detection systems are parameters extracted from traffic $[2,8,9]$ or time series $[2,3,7,10]$. The data preparation system aggregates traffic statistics over a set of specified time periods.

Mathematical methods of reducing the dimension, such as the principal component method [11] and the eigenvector method [12], are not suitable in this case. They allow one to identify the most important components in the data. For this analysis, data is taken over a significant period of time. Such mathematical methods are not applicable for operational online analysis.

Hierarchical data aggregation is used in various fields [13]. In particular, for the aggregation of time series [14]. However, this area mainly uses aggregation by characteristics, such as data source [15] or thematic aggregation [16]. Modern hierarchical aggregation in multivariate analysis does not assume the time association [17] - characteristic of self-similar processes. In turn, time aggregation [18] does not use hierarchical relationships to reduce the dimension and increase the efficiency of streaming data processing.

The general procedure for analyzing data from self-similar processes is shown in figure 1.

Thus, the task of aggregating data in the form of time series of parameters on a set of specified time intervals is particularly relevant 


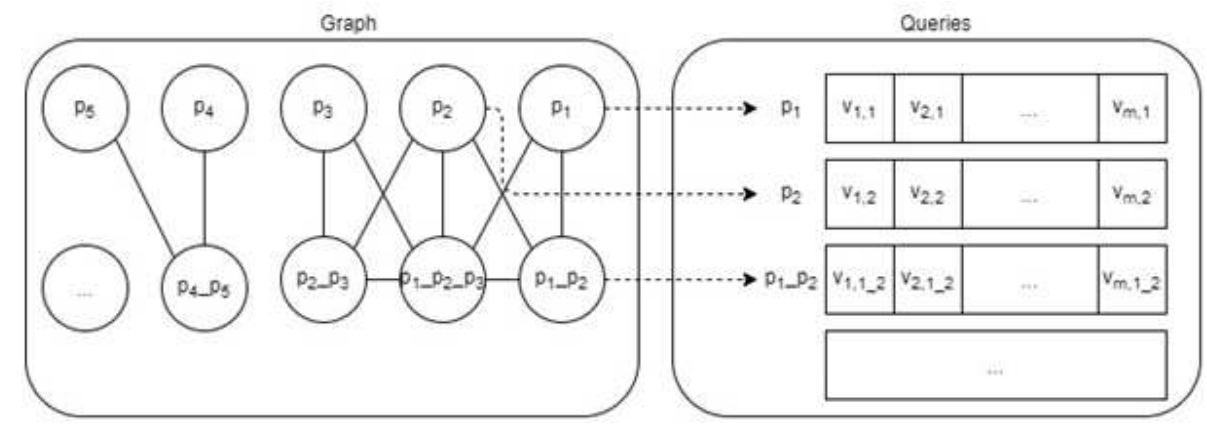

FIG. 3: Graph of relationships between queues for a single aggregation time window

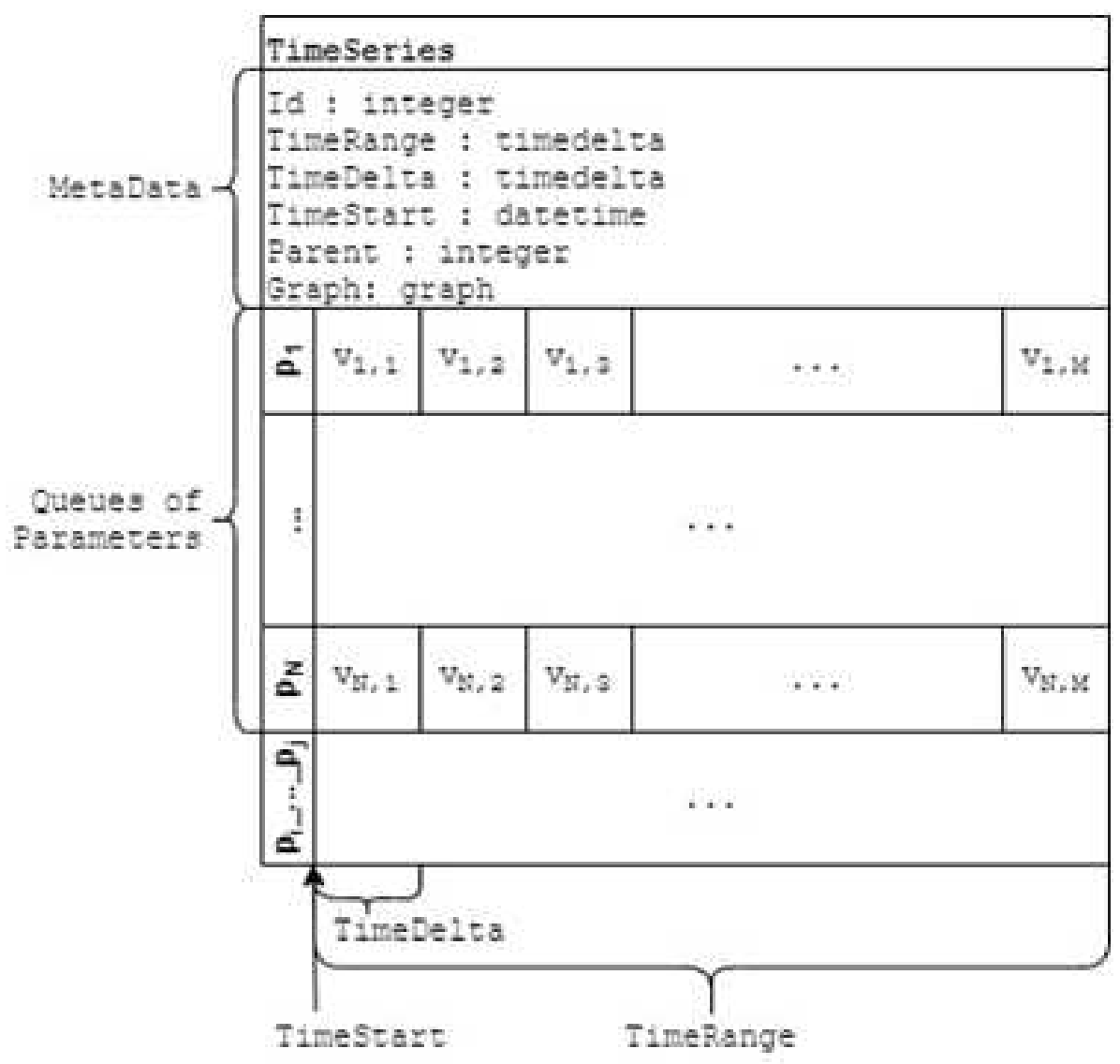

FIG. 4: Structure of the aggregation time window

for modern problems. The requirements for data aggregation for self-similar (fractal) processes analysis are:

- Universality to the type of process;

- Adaptability to changes in the number of parameters;
- Adaptability to the time depth of analysis;

- High speed;

- Small amount of data at the output;

- Suitability for streaming data processing. 
In accordance with these requirements, the authors propose aggregation methods based on the hierarchy of time segments in the analysis of self-similar processes.

\section{Hierarchical aggregation of self- similar processes data}

The approach for converting data to time series is the main one in the analysis of self-similar (fractal) processes [19]. To do this, data for a specific time period is grouped in the "aggregation time window". At the same time, each parameter $P i$ has its own queue, $Q i$, containing $\mathrm{m}$ values of this parameter (absolute or aggregated).

In order to be able to analyze data for different time periods, hierarchical relationships between time series were introduced [20]. To do this, one enter a Parent-Child Relationship between different time series and arrange for values to be shifted within each time period to get rid of old values and add new ones, as well as values to be moved from time series with a shorter time interval to time series with a longer time interval.

Before processing tuples of input data, a hierarchical structure of aggregation time windows is created. For each window, one should specify a Name, TimeRange and TimeDelta parameters, and the Parent parameter for each non-root window. The TimeStart parameter and value queues are not initialized initially and are set during the program operation. The number of queues ( $N$ value) increases dynamically during the program operation when new parameters appear in the processed data (figure 2.)

The TimeRange value must be completely divided by the TimeDelta value for the series to work correctly. The start and end values of the last element in the queue can be obtained as [TimeStart + TimeRange TimeDelta;TimeStart + TimeRange], respectively.

In this approach, the last element of the queue (the "newest" one) is incomplete, since the value is accumulated in it even before its time period is completely passed. This allows one to store data not only for time periods that have already passed, but also to have up-to-date statistics about the most recent data. There is also no need to store the current number of parent shifts and the number of parent shifts required for a new item to appear in the child window.

\section{Multidimensional}

data aggregation of self-similar processes based on related graphs

For a more complete analysis, one also need to be able to aggregate data by several parameters [7]. It is necessary not only to store the aggregated values, but also to maintain the relationships between them, to allow tracking of the relative parameters. For example, this is the percentage of packets sent between two nodes to the total number of packets sent/received by each node. The authors suggest using graphs or trees to preserve relationships and ensure that the necessary data is quickly found.

The graph structure makes it possible to calculate relative indicators in both directions or only in one direction from the child node to the parent one. The graph node is associated with aggregated data (one or more parameters). These are parameters aggregated at the appropriate level and their values.

From the point of view of implementation, if the graph stores the queues of parameters themselves, it becomes more difficult to access them. Therefore, it is more profitable, as in the hierarchical approach, to store queue lists for both single parameters and values for multiple aggregated parameters.

Access to data is provided by keys containing parameters and their values. The linked graph stores keys and the relationships between them. Thus, for each search key (parameter), one can iterate through all the nodes that it is associated with in the graph. As a result, keys will be obtained for all associated parameter queues. 
Then their relative aggregation is performed in any direction. The link structure is shown in figure 3.

Before processing tuples, a hierarchical structure of time Windows is created. For each window, one should specify a Name, the Time Range and TimeDelta parameters, and the Parent parameter for each non-root window. The Time Start parameter and value queues are not initialized initially and are set during the program operation. The Graph parameter is initially initialized with an empty graph. The number of queues (the value $N$ ) increases dynamically during the program when new parameters appear in the processed data, and decreases if the queue remains empty as a result of a time shift.

The start and end values of the last element in the queue can be obtained as [TimeStart + TimeRange TimeDelta;TimeStart + TimeRange], respectively. The TimeRange value is fixed so that it is completely divided by the TimeDelta value. The structure of the aggregation time window is shown in figure 4.

Advantages of the approach: Getting queues of required parameters is a quick procedure, since it only requires finding and retrieving the desired queue in the tree. One should store only numeric parameter queues and one graph of relationships between them for each time window. The algorithm for transferring an element from the parent window to the child window is not complicated and does not change much compared to the original approach. The last element from each queue of the parent window is moved to the first element of the child window. The parent graph is connected to the descendant graph. Different nodes may exist at different times, and at each level one can only save links for currently existing nodes.

Connections in all possible directions are saved inside the window, so a single graph is sufficient for constructing relative characteristics between any pair of parameters that have joint and separate queues.

Disadvantages of the approach: One should know in advance what parameters are used for aggregation. Introducing a new parameter requires expanding the graph structure and recalculating the relationship graph. Given the specifics of streaming data analysis, a complete reconstruction of the graph requires setting a new query over the data stream.

\section{Implementation and evaluation of the proposed method}

The developed program was tested on a dataset obtained as a result of the water treatment system [21]. The data was collected as a result of 11 days of continuous operation of the system, during which various attacks were made on the system. To implement the hierarchical structure, the anytree library is used, in particular, its NodeMixin class, which allows one to work with any class as a tree node. To implement graph storage, the networkx library and its Graph class are used. A comparison was

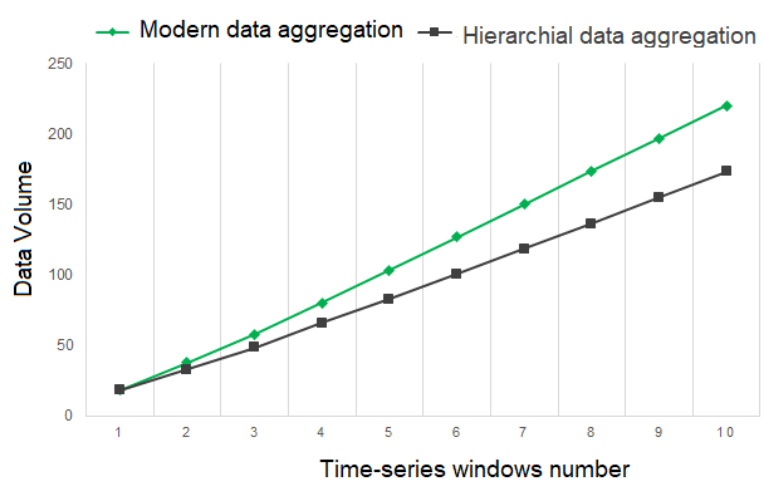

FIG. 5. (color online) Hierarchial data aggregation analysis

made of the amount of stored information for cases where hierarchical and conventional data aggregation is used (figure 5).

For multidimensional aggregation, the size and characteristics of the graph were estimated, such as the number of nodes and edges in the graph, the number of connected components, and the number of edges and nodes in the largest 
connected component. These parameters directly affect the speed of getting access to graph nodes and the speed of getting all nodes associated with the specified one. This is necessary to determine the relationships between nodes and, consequently, queues.

We analyzed the dependence of similar graph characteristics on the nesting depth of aggregated parameters (1-there are only single parameters, 2 - there are paired parameters of the form p1-p2, 3 -there are triples of parameters of the form p1p2-p3, etc.)
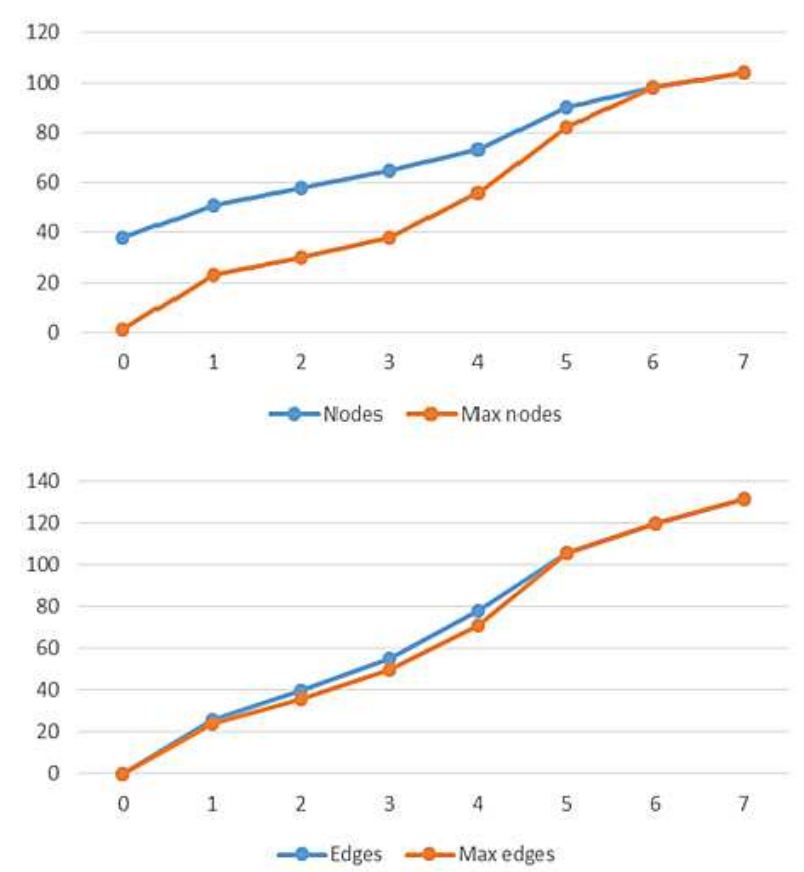

FIG. 6. (color online) Dependence of the number of nodes and edges in the graph on the number of pairs of aggregated parameters

If a pair of aggregated parameters is greater than 5 or the nesting depth of aggregated parameters is greater than 6 , the graph nodes are combined into a related component, and the efficiency of extracting parameter pairs is degraded (figures 6,7,8,9).

The graph characteristics were analyzed depending on the window time interval. During testing, the window sizes ranged from 10 seconds

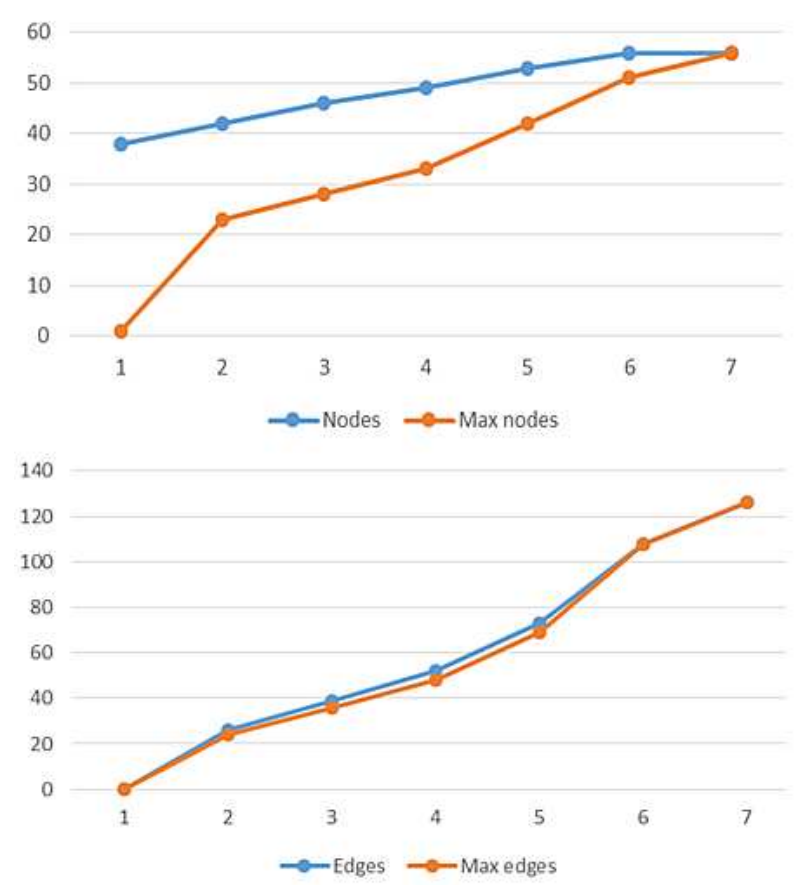

FIG. 7. (color online) Dependence of the number of nodes and edges in the graph on the depth of nesting of aggregated parameters

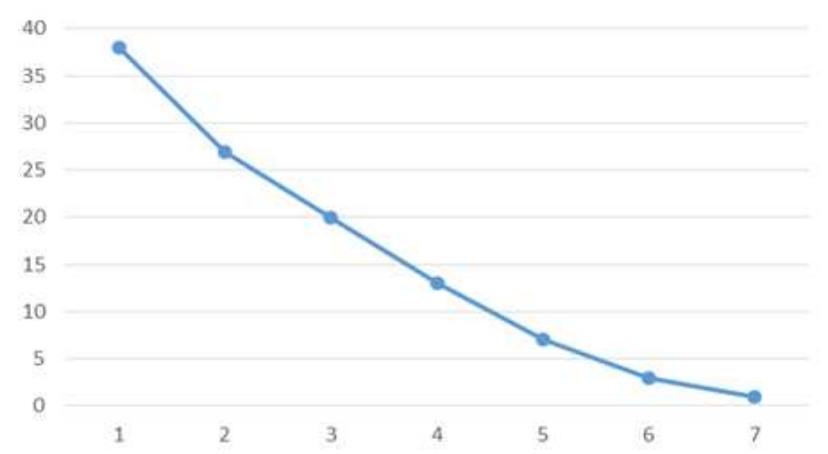

FIG. 8. (color online) Dependence of the number of connected components in the graph on the depth of nesting

to 4 hours or 14,400 seconds (see figure 10).

The additional graph requires storage space, but it is not large. The amount of data stored is about 20 bytes per vertex and / or edge. The largest graphs tested required $1 \mathrm{MB}$ of storage. Such a graph can be placed in RAM together with parameter hierarchies. 


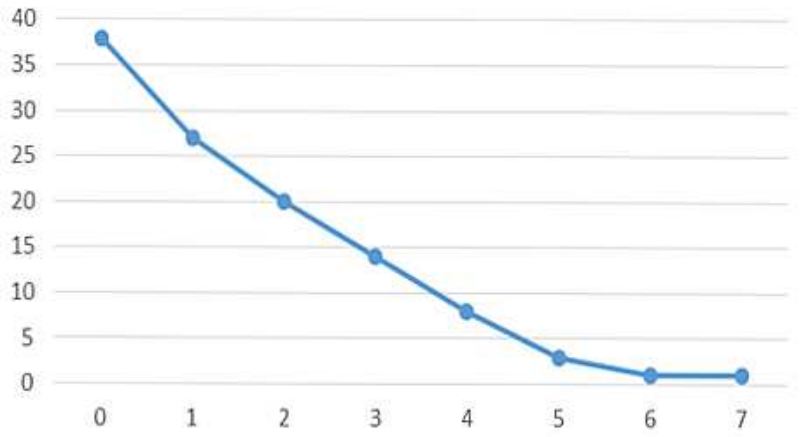

FIG. 9. (color online) Dependence of the number of connected components in the graph on the number of pairs of aggregated parameters
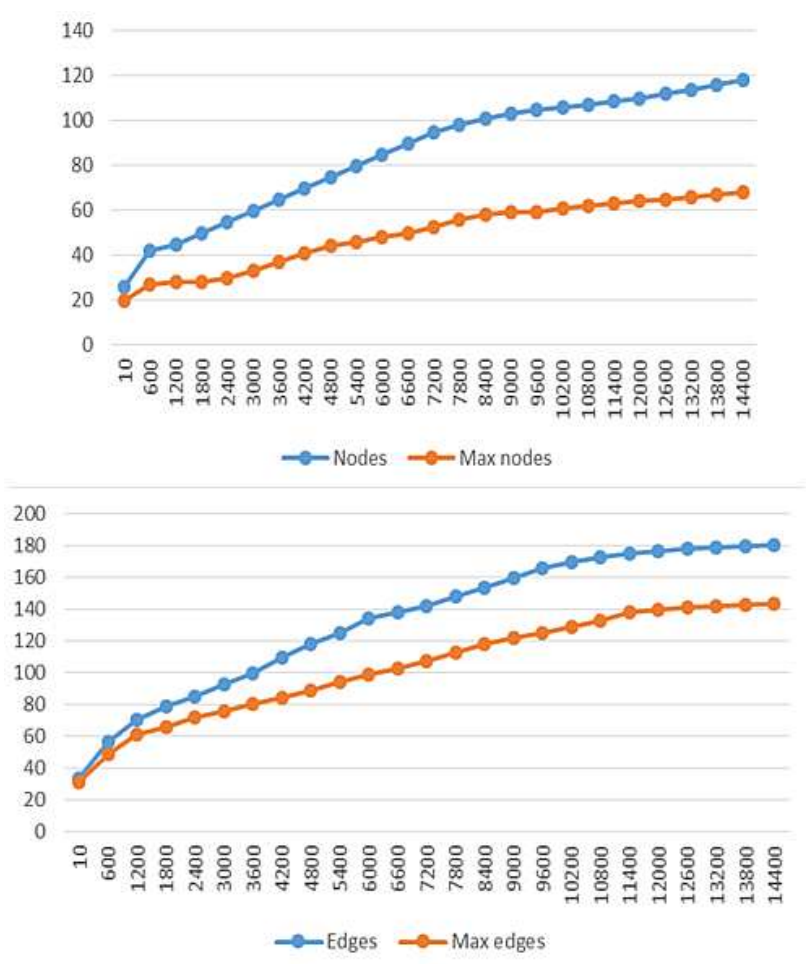

FIG. 10. (color online) Dependence of the number of nodes and edges in the graph on the window time interval (sec)

\section{Conclusion}

The introduction of a hierarchical model of aggregation time Windows increases the efficiency of data preparation for analyzing self-similar processes in data streaming. The analysis periods can range from a second to several days or months at a time. Tests have shown that using hierarchical data aggregation instead of the usual one also reduces the amount of stored information. The information for the analytical algorithm can be completely stored in RAM.

Adding multi-dimensional data aggregation across multiple parameters to the model allows one to perform more complex data analysis and use not only absolute, but also relative or correlated values without significant costs.

During testing, the boundary characteristics of the number of independent aggregated parameters and the depth of nesting of aggregated parameters for the data under consideration were revealed, as well as the value of the size of the aggregation time window, at which the graph growth slows down. These parameters depend on the original data itself, the number of unique values for each parameter, and the intensity of their receipt.

Thus, the proposed approach allows one to aggregate data when analyzing self-similar processes of various origins. The area of effectiveness of the solution is aggregation with a nesting depth of up to 5 time intervals and up to 4 aggregated parameters.

To reduce the influence of these characteristics on the graph size, one can initially fix not only the analyzed parameters, but also their values (specific nodes, specific applications, etc.). Then it is possible to determine the graph size in advance and have constant complexity in determining the dependencies between graph nodes and queues.

\section{Acknowledgments}

The reported study was funded by Russian Ministry of Science (information security), project number $2 / 2020$. 


\section{References}

[1] W. E. Leland, M. S. Taqqu, W. Willinger, and D. V. Wilson, IEEE-ACM Transactions on Networking 2, 1 (1994).

[2] O. Sheluhin, S. Smolskiy, and A. Osin, Selfsimilar processes in telecommunications (John Wiley and Sons, 2007).

[3] D. Lavrova, I. Alekseev, and A. A.A. Shtyrkina, Automatic Control and Computer Sciences 52, 931 (2018).

[4] M. Kleppmann, Designing Data-Intensive Applications: The Big Ideas Behind Reliable, Scalable, and Maintainable Systems (O-Reilly Media, Boston, 2016).

[5] D. Lavrova, M. Poltavtseva, and A. Shtyrkina, in 2018 IEEE Industrial Cyber-Physical Systems (ICPS) (IEEE, St. Petersburg, 2018), pp. 818823.

[6] G. Mazzini, R. Rovatti, and G. Setti, IEICE TRANSACTIONS on Fundamentals of Electronics, Communications and Computer Sciences E88-A, 2656 (2005).

[7] D. Zegzhda, D. Lavrova, and A. Khushkeev, in IEEE International Conference on Industrial Cyber Physical Systems (ICPS) (IEEE, Taipei, Taiwan, 2019), pp. 780-784.

[8] P. Zegzhda, D. Zegzhda, E. Pavlenko, and A. Dremov, in 10th International Conference on Security of Information and Networks (SIN $65^{T M} 17$ ) (Association for Computing Machinery, New York, 2017), pp. 280-в Ђ“283.

[9] M. Kalinin and E. Pavlenko, Automatic Control and Computer Sciences 49, 673 (2015).

[10] A. Bar, A. Finamore, P. Casas, L. Golab, and M. Mellia, in 2014 IEEE International Conference on Big Data (Big Data) (IEEE, 2014), pp. 165-170.

[11] K. Pearson, Philosophical Magazine 2, 559в Ђ" (1901).

[12] G. Golub and C. V. Loan, Matrix Computations (Johns Hopkins University Press, Baltimore, 1996).

[13] H. Wang and H. Chen, in 11th International
Conference on Wireless Communications and Signal Processing (WCSP) (IEEE, Xian, China, 2019), pp. 1-в Ђ“6.

[14] P. Furtado, Euro-Par 2005. Lecture Notes in Computer Science 3648, 360 (2005).

[15] D. Hughes and P. Singh, Hierarchical aggregation of select network traffic statistics, Silver Peak Systems Inc, https://patents.google.com/patent/ patent us20200021506a1 (2020).

[16] M. Leonard, K.E.Crowe, S.M.Christian, J. S. Beeman, D.B.Elsheimer, and E. Blair, Computer-implemented systems and methods for efficient structuring of time series data, SAS Institute Inc, https://patents.google.com/patent/US9244887B2, patent us9244887b2 ed. (2016).

[17] E. Williamson, Systems and methods for hierarchical aggregation of multidimensional data sources, Red Hat Inc, https://patents.google.com/patent/US8495007B2/, patent us8495007b2 ed. (2013).

[18] R. Bakalash, G. Shaked, and J. Caspi, System with a data aggregation module generating aggregated data for responding to OLAP analysis queries in a user transparent manner, Intellectual Ventures Assets 114 LLC, Mec Management LLC, https://patents.google.com/patent/US8170984B2, patent us8170984b2 ed. (2012).

[19] O. Antipov, degree of doctor of physical and mathematical sciences, Volga State University of Telecommunications and Informatics, Russian Federation, Samara (2011).

[20] M. A. Poltavtseva, P. D. Zegzhda, and I. D. Pankov, in 2018 Eleventh International Conference Management of large-scale system development (IEEE, 2018), pp. 1-5.

[21] J. Goh, S. Adepu, K. Junejo, and A. Mathur, Critical Information Infrastructures Security. Lecture Notes in Computer Science 10242, 1 (2016). 